\title{
Grasping partly occluded objects: Effects of global stimulus information on action
}

\author{
Janneke Lommertzen, Rob van Lier, Ruud G J Meulenbroek \\ Donders Institute for Brain, Cognition and Behaviour, Radboud University Nijmegen, PO Box 9104, \\ 6500 HE Nijmegen, The Netherlands; e-mail: janneke.lommertzen@googlemail.com \\ Received 20 May 2008, in revised form 12 August 2008
}

\begin{abstract}
We investigated here how partly occluded stimuli affect early and late prehension kinematics. Stimuli were pictures of partly occluded cylindrical objects with indentations or protrusions at regular intervals along the contour. They diverged into two plausible completions: a local completion, comprising a linear continuation of the contour, and a global completion, revealing a continuation of the contour indentations. Convergent stimuli with one plausible completion acted as controls. Twenty-eight participants repeatedly performed a grasping movement in darkness, as if they grasped the earlier-seen partly occluded cylinder. The peak acceleration of the hand opening (occurring at about $20 \%$ of the movement time) and the maximum hand aperture (at about $60 \%$ of the movement time) were taken to reflect the representation of the width of the stimuli used for action. The results show that the grasping movements were affected not only by local but also by global stimulus properties of which the representation remained stable over time.
\end{abstract}

\section{Introduction}

We act on and manipulate numerous objects each day but we never act on completely visible objects. Parts of objects are often hidden behind protruding elements of the object itself or by other objects. Imagine a carton of juice on a full breakfast table. Its base and rear side may not be visible because of the viewing angle and other parts of the carton may be hidden from view owing to the presence of other intermediate objects on the table. Despite all this, we don't experience the carton as being fragmented or incomplete. Furthermore, we are perfectly able to pick up the carton to pour out some juice.

The present study aims at a better understanding of the ways in which the action system copes with fragmented visual information when participants are asked to grasp partly occluded objects. Objects protruding from behind an occluder are interpreted as one whole object when their contours are relatable (eg Kellman and Shipley 1991) and their volumes are mergeable (Tse 1999b). This so-called amodal completion process takes multiple steps and starts with a representation of the visible physical features early in the visual cortex and ends with a representation of a completed shape in higher visual areas (Murray et al 2006; Weigelt et al 2007). Partly occluded objects can be interpreted in an infinite number of ways; however, some solutions are more likely than others. Other studies of amodal completion have shown that different strategies lead to different stimulus interpretations (van Lier et al 1995a, 1995b; Sekuler 1994; Sekuler et al 1994; de Wit et al 2006; de Wit and van Lier 2002). Whereas global completions take into account various overall characteristics of the whole object, like symmetries or repetitions (van Lier et al 1994; de Wit et al 2006; de Wit and van Lier 2002), local completions are derived from contour information that is available at or near the points of occlusion. In particular, local completions are generally formed by a linear or curvilinear continuation of the partly occluded contour behind the occluder (eg Fantoni and Gerbino 2003; Kellman and Shipley 1991; Singh and Fulvio 2005; Wouterlood and Boselie 1992). Van Lier et al (1995a, 1995b) have argued that the preference for one of the two 
types of completion results from a competition between interpretations. Patterns with stronger competition between different possible interpretations are more ambiguous.

Thus far, completion has mostly been studied in perceptual experiments in which observers had to judge the form of the partly occluded shape by responding to different visual solutions (Gerbino and Salmaso 1987; van Lier et al 1995b; van Lier and Wagemans 1999; Tse 1999a; de Wit and van Lier 2002). Here, we turn to a more complex behavioural response. In real-life situations we act upon objects around us, and we may grasp them at parts that are occasionally blocked from sight. In fact, we often grasp earlier-seen objects without direct visual control. The remembered object position and object shape normally suffice to perform a grasping movement successfully. In the present study, we therefore asked participants to perform grasping movements to earlier-seen partly occluded objects. We focused on how our stimuli would affect an early and a late kinematic feature of the grasping movements because we presumed that the representations of the occluded cylinders, specifically the more complex ones, might become less accurate with time.

We designed two types of partly occluded shapes as stimuli: not ambiguous ones and ambiguous ones (figure 1). To create these stimuli we accounted for local and global completion strategies (eg van Lier et al 1995a, 1995b; van Lier and Wagemans 1999; Sekuler 1994; Sekuler et al 1994; de Wit et al 2006). Stimuli of the first type, referred to here as 'global-local convergent', were designed such that local and global strategies converged to the same solution, whereas those of the second type, referred to as 'global-local divergent', were designed such that local and global strategies diverged into different completions. We tested the perceptual ambiguity of our stimuli in a control

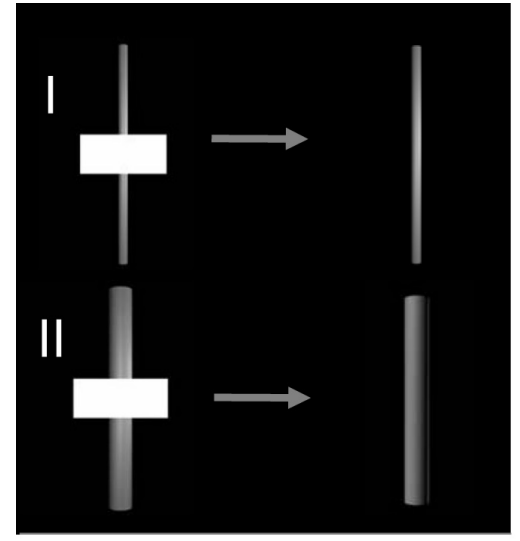

(a)

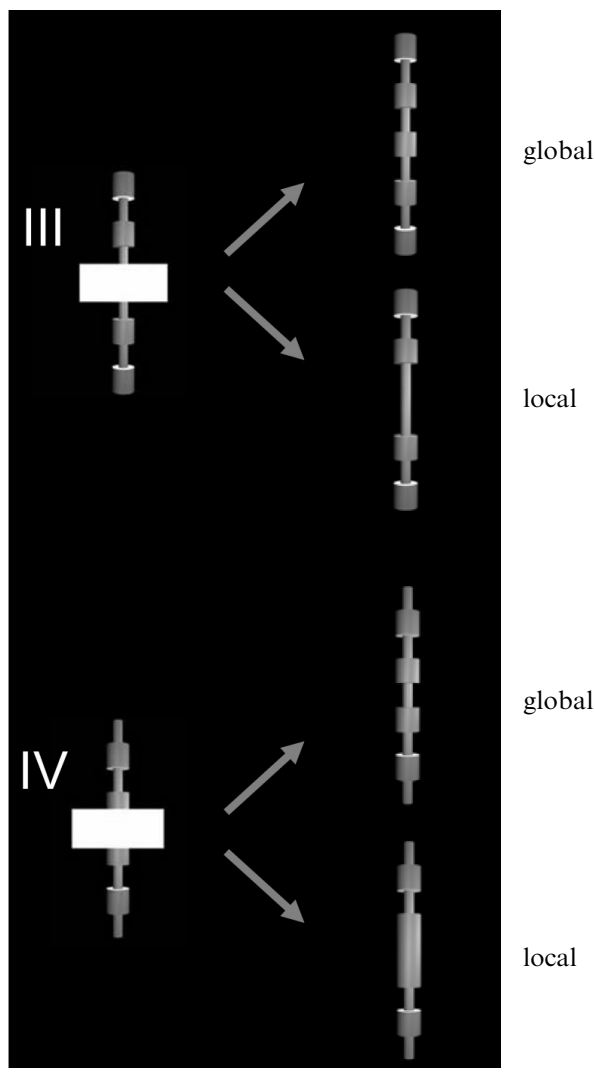

(b)

Figure 1. Occluded stimuli and their completions: (a) the convergent stimuli; (b) the divergent stimuli. The completions (on the right) of stimuli I and II represent both a global and a local completion, whereas the completions of stimuli III and IV can be global or local. 
experiment in which we exploited a simultaneous-matching paradigm. To reveal whether our perceptually ambiguous stimuli would differentially affect early or late prehension kinematics, we analysed two different kinematic measures. The first measure was the amplitude of the first peak of the aperture acceleration profile (aApt), which occurs at about $20 \%$ of the movement time and can be regarded as a relatively early indicator of the grasping response that was planned. The second measure was the maximum grip aperture (MApt), which occurs generally at about two-thirds of the movement time, and which can be regarded as a relatively late indicator of the planned grasping response (Jeannerod 1984; Marteniuk et al 1990; Meulenbroek et al 2001; Smeets and Brenner 1999). In terms of Glover's (2004) distinction between planning and control, we assumed that the former index would primarily reflect planning processes and the latter potentially additional on-line control processes on the basis of, for example, haptic feedback processes. According to Glover (2004), the influence of both these processes will rise and fall as the movement unfolds. Peak grip acceleration, a sizedependent parameter that occurs at roughly $35 \%$ of movement duration (Jakobson and Goodale 1991; Jeannerod 1984), will reflect planning more than control, whereas maximum grip aperture will reflect control more than planning (Glover 2004).

Only if the thin cylinder leads to a smaller maximum grip aperture and the thick one to a larger maximum grip aperture could we be sure that the size information presented by us to the participants was reflected in their grasping movements. If participants used a local completion strategy we would find similar MApt's and aApt's for stimuli I and III, and also for stimuli II and IV. Local completion of the occluded parts of stimuli I and III would lead to representations with identical thin central segments and local completion of the occluded parts of stimuli II and IV would lead to representations with identical thick central segments (see figure 1). However, if subjects adopted a global completion strategy we would expect the grasping movements to show similar MApt's and aApt's in response to stimuli I and IV, and also in response to stimuli II and III.

\section{Grasping experiment}

\subsection{Method}

2.1.1 Participants. Twenty-eight participants, four male and twenty-four female, with ages ranging from 18 to 29 years (mean age 23 years) participated in a grasping task after giving their informed consent. All participants performed the grasping movements with their preferred, right hand.

2.1.2 Material and design. The participants were asked to repeatedly grasp, in darkness, a cylinder with a fixed diameter. All grasping movements involved a precision grip, ie the participants were instructed to grasp the target cylinder with the tips of the thumb and fingers rather than the palm of the hand touching the cylinder surface. The to-be-grasped cylinder remained the same throughout the experiment, but the stimuli of the partly occluded cylinders that we showed our participants before each grasp varied.

Stimuli consisted of pictures of 3-D cylinders and cylinder-like objects with a length of $\sim 37.5 \mathrm{~cm}(8.8 \mathrm{deg}$ visual angle) and a diameter of $1.4 \mathrm{~cm}$ or $2.8 \mathrm{~cm}(0.33$ or $0.66 \mathrm{deg}$, respectively; see figure 1). The centres of the stimuli were occluded by a $6.4 \mathrm{~cm} \times 16.1 \mathrm{~cm}(1.5 \mathrm{deg} \times 3.8 \mathrm{deg})$ white rectangle. We used two types of occluded stimuli: stimuli that consisted of simple cylinders, and more complex ones with indentations or protrusions along the contour. Stimuli were designed such that with the first type of stimuli, the so-called global-local convergent stimuli, both local and global completion strategies converged to the same interpretation (figure 1, stimuli I and II), and with the latter, more complex global-local divergent stimuli, local and global completion strategies diverged to different interpretations (figure 1, stimuli III and IV). 
The local completion results from a linear continuation of the contours at the points of occlusion, whereas the global completion takes account of the sequence of indentations and protrusions in the visible parts of the cylindrical object. Our stimuli are related to the partly occluded 2-D shapes with indentations and protrusions that were used in a MEG study by de Wit et al (2006), which demonstrated that for the control stimuli, both global and local completions converged to the same interpretation, whereas for the experimental stimuli, global and local completions diverged to different interpretations.

Participants sat comfortably at a black table, on a height-adjustable chair with armrests. A projection screen stood on the table at a distance of $243 \mathrm{~cm}$ from the participant. Stimuli were projected on the backside of the screen with a Sharp, $60 \mathrm{~Hz}$ LCD projector. A target cylinder (diameter $\times$ height: $3.3 \mathrm{~cm} \times 20 \mathrm{~cm}$ ) stood on the table within reaching distance at about $40 \mathrm{~cm}$ in front of the body midline of the participant. A small piece of sandpaper indicated the starting position for the right hand with which the grasps had to be generated. It was stuck to the edge of the table at about $20 \mathrm{~cm}$ to the right from the participant's body midline.

Two Optotrak 3020 (Northern Digital, Waterloo, Canada) camera units recorded the grasping movements during $2 \mathrm{~s}$, from the 'go' signal until the end of the trials, with a sampling frequency of $100 \mathrm{~Hz}$. Kinematics were deduced from three infrared emitting diodes (IREDs) that were attached to the wrist (processus styloideus radius) and the tips of index finger and thumb.

2.1.3 Procedure. After having received written and verbal instructions, participants performed several practice trials to familiarise themselves with the task and to test whether all IREDs could be detected by the Optotrak system. After this, each participant performed four blocks of 80 trials, each trial block lasting about $10 \mathrm{~min}$, with short breaks in-between. The room was darkened during each trial block to prevent subjects from seeing the to-be-grasped cylinder. After every block the lights were switched on to prevent subjects from completely adjusting to the darkness.

A stimulus was shown for $500 \mathrm{~ms}$, after which a high-frequency acoustic 'go' signal was presented after a delay of $17 \mathrm{~ms}$ or $1000 \mathrm{~ms}$. The short delay was used to put a time stress on the amodal completion process that we assumed would follow stimulus presentation. The long delay was chosen under the assumption that it would allow assessing any effects of the representations becoming less accurate as a function of time. Participants were instructed to grasp the target cylinder as if they were grasping the cylindrical object that was previously projected, in the centre, ie the part behind the occluder. After the $2 \mathrm{~s}$ recording period was finished, a low-frequency tone sounded indicating the end of a trial. In-between trials participants had to grasp a 'recalibration bar' between the thumb and index finger before returning to the start position to wait for the next trial (see figure 2).

Even though participants were asked to repeatedly grasp one and the same cylindrical object, we took two precautionary measures to ensure that they responded to the stimulus instead of using a response strategy like performing the same grasping movement in every trial. Our first measure to make sure that participants responded to the stimuli was to vary the diameter of a 'recalibration bar', a cylinder with a diameter of either $1.3 \mathrm{~cm}$ or $5.3 \mathrm{~cm}$ that was attached to the table in a horizontal position, aligned with the participant's sagittal plane, at approximately $20 \mathrm{~cm}$ to the right of the participant's right shoulder. The recalibration bar was used to mask the haptic feedback from the target cylinder. Participants grasped this recalibration bar in-between trials without vision and, by doing this, they got haptic feedback from a cylinder with a diameter that was either $2 \mathrm{~cm}$ smaller or $2 \mathrm{~cm}$ larger than the target cylinder. 


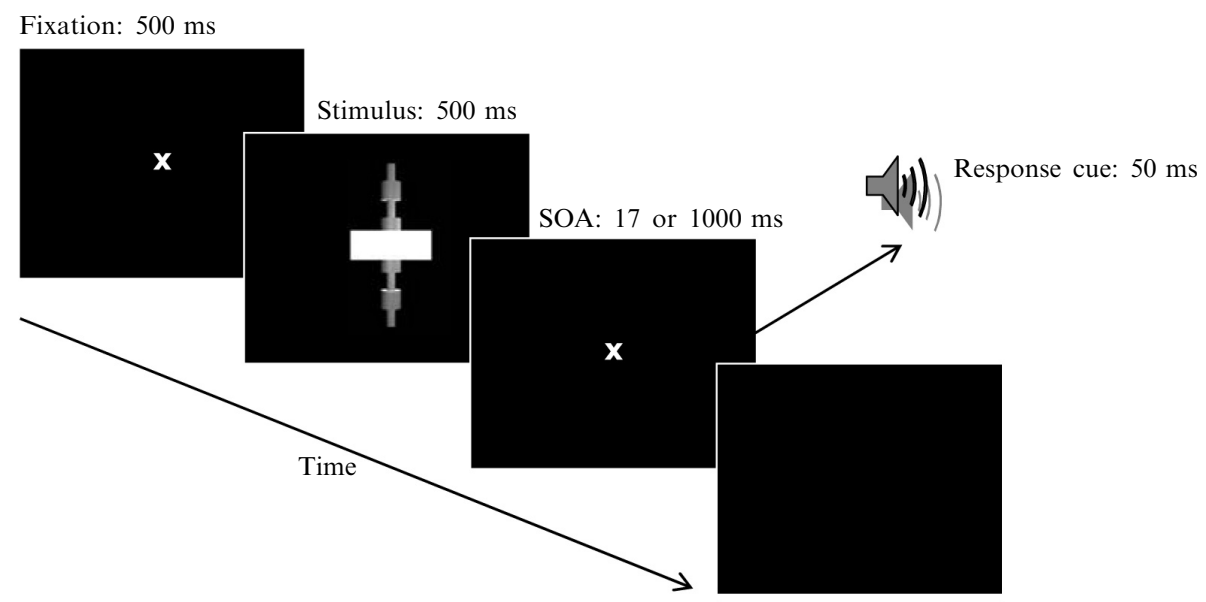

Top view of table

Figure 2. Trial events in the grasping task. Note that the grasping of the recalibration bar between trials is not depicted.

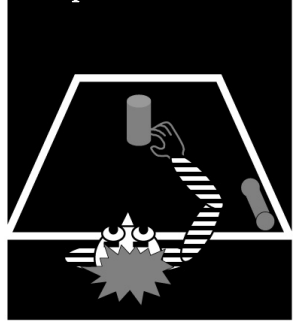

The second measure to make sure that participants responded to the stimuli was to verbally prime participants to grasp the narrow cylinder in half of the trials and to grasp the wide cylinder in the other half of the trials. This was accompanied by varying the relative proportion of convergent trials with a small-diameter stimulus (figure 1, stimulus I) and a large-diameter stimulus (stimulus II) between blocks. All stimuli were shown in every block. The number of trials with divergent stimuli III and IV remained constant across blocks (20 trials with each divergent stimulus), whereas the number of trials with convergent stimuli I and II differed. This way, in half the blocks, stimulus I was shown 10 times out of 80, and stimulus II 30 times; for the other half of the blocks this ratio was reversed. Both the priming and the masking measures were counterbalanced across conditions.

2.1.4 Data analysis. The IRED-position data were filtered with a dual-pass, secondorder, low-pass Butterworth filter with a cut-off frequency of $8 \mathrm{~Hz}$. Missing data were linearly interpolated. The start and the end of the responses were defined as the first and the last local minimum in the tangential velocity function of the IRED positioned on the wrist, where the velocity reached a threshold value of $5 \%$ of the peak velocity in that trial. Subsequently, in each trial the maximum grip aperture (MApt in $\mathrm{cm}$ ) was determined. Then, time-normalised velocity and acceleration profiles of the grip aperture were computed. The first peak of the aperture acceleration (aApt in $\mathrm{cm} \mathrm{s}^{-2}$ ) that exceeded $10 \%$ of the maximum acceleration of that trial was taken as an early index reflecting the planned grasping response, based on the interpreted diameter of the occluded stimulus part. We derived this variable by means of a semi-automatic Matlab procedure, which involved visual inspection of the kinematics of every trial. To grasp a larger object, a larger initial aApt (and MApt) is needed than for grasping smaller objects. With this in mind, we deduced whether a local or a global interpretation of the stimuli was used to perform the grasping responses. This was evaluated by means of an ANOVA with one factor (stimulus IV), and a posteriori $t$-tests for aApt and MApt. 
To obtain a general measure for the individual completion preference (local or global) of each participant, we computed the difference between the aApt of grasps in response to stimulus III and the aApt of grasps in response to stimulus IV. If the grasping responses were executed and guided on the basis of a stable perceptual representation, then both aApt(stim III - stim IV) and MApt(stim III - stim IV) would reflect the same completion preference for every participant, ie have the same sign for every participant. To check whether the completion preferences we found by this method were not confounded by differences in the variability of aApt and MApt within subjects, we computed the coefficient of variance $(\mathrm{CoV})$ per participant according to equation (1). ${ }^{(1)}$ A higher $\mathrm{CoV}$ would imply a larger within-subject variability. Only if the CoVs are equally distributed across participants, can we be sure that the (stim III - stim IV) differences are not due to extreme variability in the response pattern of the participants with the most pronounced local or global response patterns. Both extremely variable completion preference and grasping behaviour and very stable responses based on an averaged completion preference can result in aApt(stim III - stim IV) and MApt(stim III - stim IV) close to zero. In the statistical analyses in this and the following experiment we used an $\alpha$ of 0.05 .

$$
\mathrm{CoV}=\frac{\mathrm{SD}[\mathrm{MApt}(\operatorname{stim} \mathrm{III})]+\mathrm{SD}[\mathrm{MApt}(\operatorname{stim} \mathrm{IV})]}{\operatorname{mean}[\operatorname{MApt}(\operatorname{stim} \text { III })]+\operatorname{mean}[\operatorname{MApt}(\operatorname{stim} \mathrm{IV})]}
$$

2.1.5 Exclusion criteria. If five or more frames of one or more IREDs were missing, the data were discarded, which was the case in $17.3 \%$ of all trials. If fewer than five frames were missing, position data were linearly interpolated. In addition, responses that had started before the response cue and responses that ended too late $(\mathrm{RT}+\mathrm{MT}>2 \mathrm{~s})$ were also excluded from the analyses. RT was defined as the duration of the interval between the 'go' signal and the start of the response, and MT was defined as the duration of the interval between movement onset and the end of the response. Trials with MApt or aApt that differed more than three standard deviations from mean were considered outliers. On the basis of this criterion, trials with a MApt $>11.3 \mathrm{~cm}$, and trials with an aApt $>457 \mathrm{~cm} \mathrm{~s}^{-2}$ were excluded from further analysis. As a result, another $4.1 \%$ of the trials were excluded.

\subsection{Results}

The results were not different for the 17 and $1000 \mathrm{~ms}$ delay conditions $\left(t_{27}=1.526\right.$, ns; and $t_{27}=1.801$, ns, for MApt and aApt, respectively), and the data were therefore pooled across this factor. We will return to the absence of any effect of the time-delay factor in section 4 .

Figure 3 shows the time-normalised grasping kinematics for responses to the four different stimuli, averaged across all participants. Convergent and divergent stimuli are plotted in separate panels. The aperture-time functions are plotted in the top panels, and the acceleration profiles are plotted in the bottom panel.

Mean MApt was $7.7 \mathrm{~cm}(\mathrm{SE}=0.22 \mathrm{~cm})$, and MApts for stimuli I, II, III, and IV were $7.5 \mathrm{~cm}(\mathrm{SE}=0.25 \mathrm{~cm}), 7.9 \mathrm{~cm}(\mathrm{SE}=0.24 \mathrm{~cm}), 7.7 \mathrm{~cm}(\mathrm{SE}=0.22 \mathrm{~cm})$, and $7.8 \mathrm{~cm}$ $(\mathrm{SE}=0.24 \mathrm{~cm})$, respectively. A one-way repeated-measures ANOVA showed an effect of stimulus $\left(F_{3,81}=3.234, p<0.05\right)$. A posteriori paired samples $t$-tests showed a significantly larger MApt in trials with stimulus II than in trials with stimulus I $\left(t_{27}=2.330, p<0.05\right)$, thus confirming the sensitivity of our paradigm. MApt did not differ between the divergent stimuli $\left(t_{27}<1\right.$, ns). Furthermore, the differences between

(1) It could be argued that the $\mathrm{CoV}$ should be computed by dividing the difference between the SDs by the difference between the means. However, when the difference between the means reaches zero, this would increase the $\mathrm{CoV}$ disproportionally. For this reason, we decided to compute the $\mathrm{CoV}$ using the sum of SDs divided by the sum of means. 

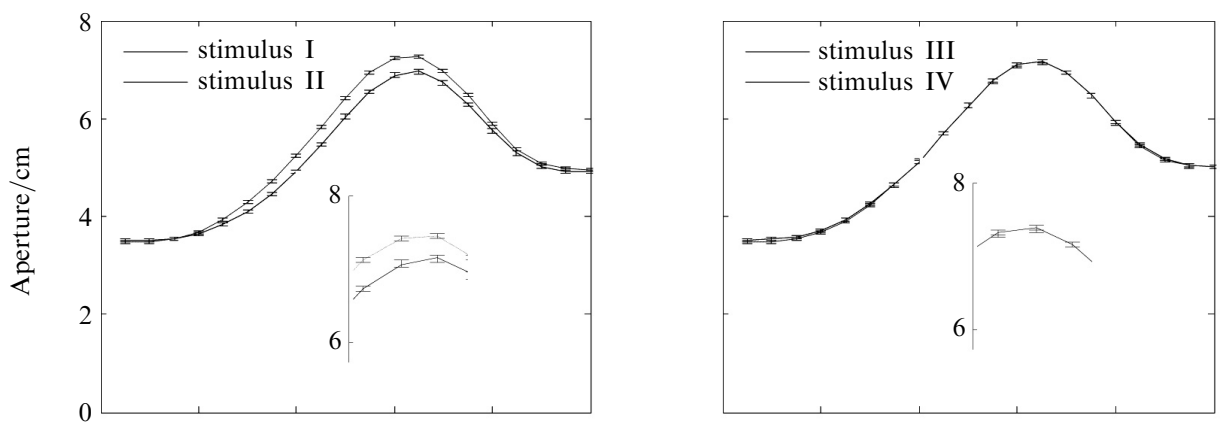

(a)

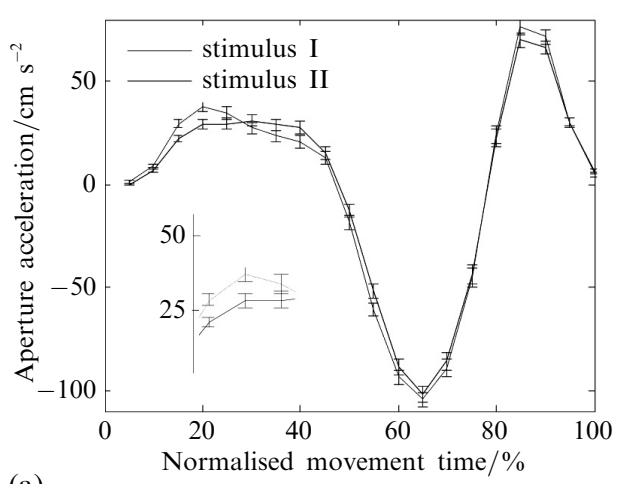

Figure 3. Time-normalised grasping kinematics for convergent (a) and divergent (b) stimuli, averaged across all participants. The top row shows the aperture and the bottom row shows the aperture acceleration. The inserts in each panel show enlargements of the important peaks of the aperture and acceleration profiles.

stimuli I and III $\left(t_{27}=2.261, p<0.05\right)$, and between stimuli II and IV $\left(t_{27}=2.680\right.$, $p<0.05)$ were statistically significant, whereas there were no differences between stimuli II and III $\left(t_{27}=1.533\right.$, ns), and between stimuli I and IV $\left(t_{27}=1.523\right.$, ns), which reflects the influence of global stimulus properties.

The difference between MApt for stimulus II and stimulus IV ranged from $-3.32 \mathrm{~cm}$ to $1.16 \mathrm{~cm}$ (mean $=-0.03 \mathrm{~cm}, \mathrm{SE}=0.13 \mathrm{~cm}$ ), as shown in figure 4. As mentioned before, we computed $\mathrm{CoV}$ per participant in order to get an impression of the within-subjects variability of MApt and aApt. Across the participants, CoV for MApt ranged from 0.06 to $0.17 \mathrm{~cm}$ (mean $=0.10 \mathrm{~cm}$; see lower panel of figure 4 ), which demonstrates that the between-subjects variation in MApt was larger than the within-subjects variation.

The average amplitude of the first peak of aApt was $150.1 \mathrm{~cm} \mathrm{~s}^{-2}\left(\mathrm{SE}=13.3 \mathrm{~cm} \mathrm{~s}^{-2}\right)$ and the values of aApt for responses to stimuli I, II, III, and IV were $144.5 \mathrm{~cm} \mathrm{~s}^{-2}$ $\left(\mathrm{SE}=13.2 \mathrm{~cm} \mathrm{~s}^{-2}\right), 155.9 \mathrm{~cm} \mathrm{~s}^{-2}\left(\mathrm{SE}=14.0 \mathrm{~cm} \mathrm{~s}^{-2}\right), 148.4 \mathrm{~cm} \mathrm{~s}^{-2}\left(\mathrm{SE}=13 \mathrm{~cm} \mathrm{~s}^{-2}\right)$ and $151.4 \mathrm{~cm} \mathrm{~s}^{-2}\left(\mathrm{SE}=14.3 \mathrm{~cm} \mathrm{~s}^{-2}\right)$, respectively (one way ANOVA: $F_{3,81}=2.000, p=0.12$ ). Note that the peak aApt values shown in figure 3 are the result of having averaged time-normalised aperture acceleration-time functions whereas the average peak aApt values listed here were obtained by averaging the peak grasp-acceleration values observed in the first phase of the grasping movements irrespective of the (relative) moment at which they occurred. Since aApt peak values could occur at any moment between $15 \%$ and $35 \%$ of MT, their peak value of, on average, $150 \mathrm{~cm} \mathrm{~s}^{-2}$ dropped to around $50 \mathrm{~cm} \mathrm{~s}^{-2}$ as a result of averaging across trials. Paired samples $t$-tests showed a marginal difference between aApt in trials with stimuli I and II $\left(t_{27}=1.861, p=0.074\right)$, and no difference between stimuli III and IV $\left(t_{27}<1\right.$, ns). 

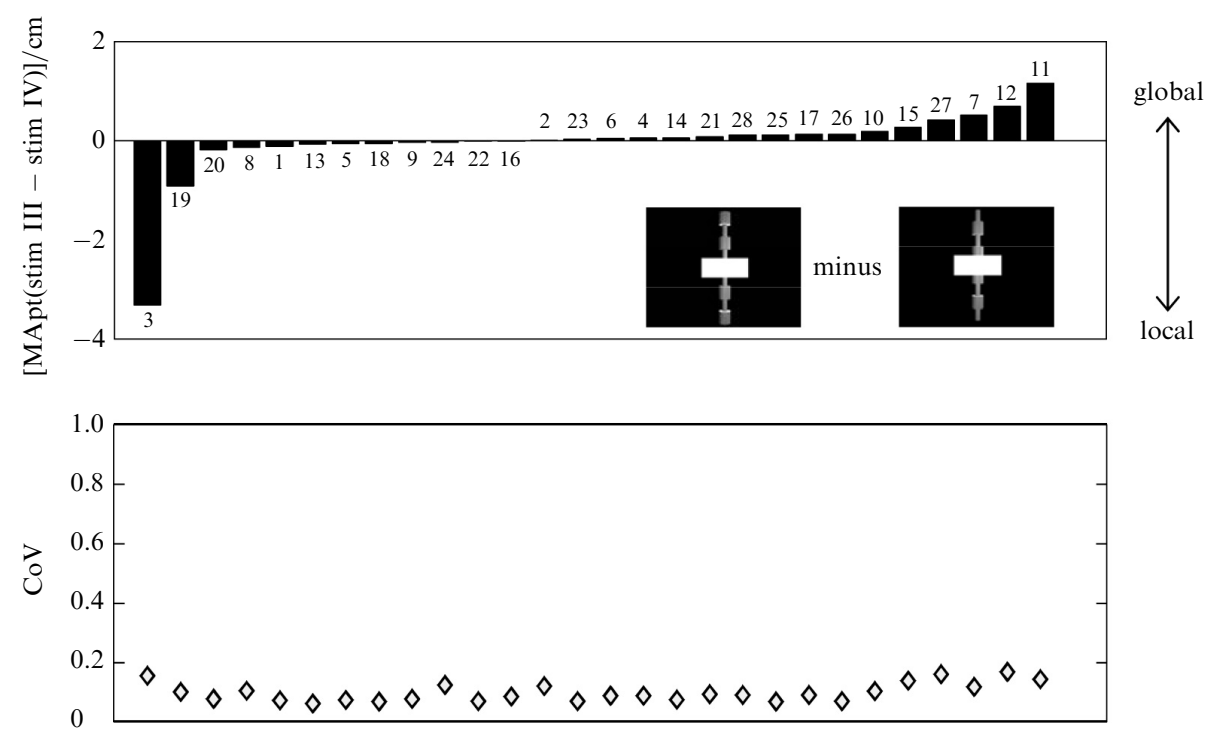

Figure 4. Maximum aperture (MApt) differences between responses to stimulus III and stimulus IV for each participant are shown in the top panel. Participant numbers are depicted under and above the bars. The lower panel shows the corresponding coefficients of variance $(\mathrm{CoV})$ for every participant.

As for MApt, we also computed the aApt difference and $\mathrm{CoV}$ for each participant: aApt(stim III) - aApt(stim IV) ranged from $-55.5 \mathrm{~cm} \mathrm{~s}^{-2}$ to $65.8 \mathrm{~cm} \mathrm{~s}^{-2}$ $\left(\right.$ mean $=-3.0 \mathrm{~cm} \mathrm{~s}^{-2}, \mathrm{SE}=4.7 \mathrm{~cm} \mathrm{~s}^{-2}$ ), and $\mathrm{CoV}$ for aApt ranged from $0.34 \mathrm{~cm} \mathrm{~s}^{-2}$ to $0.96 \mathrm{~cm} \mathrm{~s}^{-2}$ (mean $=0.58 \mathrm{~cm} \mathrm{~s}^{-2}$ ); both are shown in figure 5. Again, the CoV of the aApt data confirmed that the between-subjects variation in aApt was larger than the withinsubjects variations in MApt.
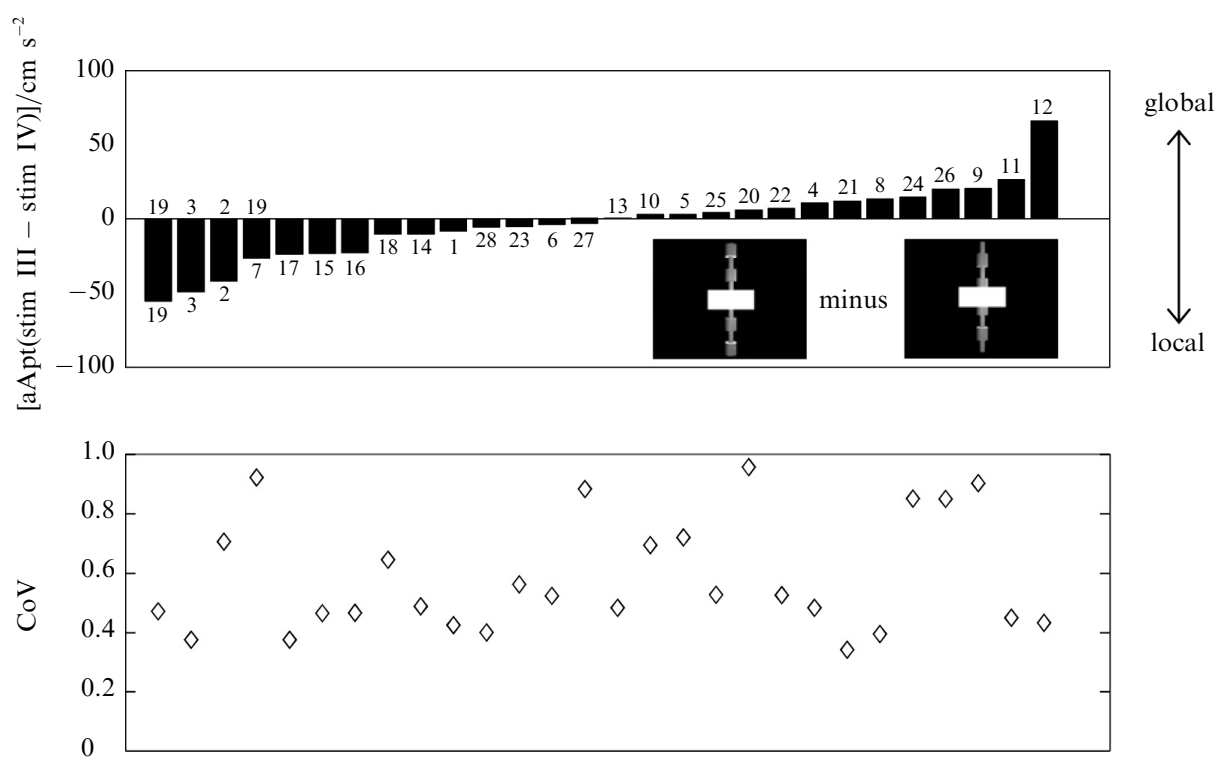

Figure 5. Aperture acceleration (aApt) differences between responses to stimulus III and stimulus IV for each participant are shown in the top panel. Participant numbers are depicted under and above the bars and the lower panel shows the corresponding coefficients of variance $(\mathrm{CoV})$ for every participant. 
The relationship between the aApt and MApt was analysed by computing the correlations between these two measures for every stimulus. In all cases there was a positive correlation between the early and late kinematic measures. Pearson correlation coefficients $(N=28)$ for the convergent stimuli were significant for both stimulus I $(r=0.466, p<0.05)$ and stimulus II $(r=0.588, p<0.01)$, as were the correlation coefficients for the relationship between aApt and MApt in responses to the divergent stimuli III $(r=0.400, p<0.05)$ and IV $(r=0.637, p<0.01)$. Correlations between the two kinematic measures, computed for each participant, and for every stimulus separately, were positive and significant $(p<0.05)$ for all but one participant.

The correlation between the MApt difference for stimuli III and IV (see figure 4) on the one hand and the aApt difference for these stimuli on the other (see figure 5), determined to compare the completion preference early and late in the responses for each participant separately was also positive $(r=0.546, p<0.01)$. Comparing $\mathrm{CoV}$ for both measures, it is clear that aApt had a larger variability. The bottom panels of figures 4 and 5 show that this variability was unrelated to the difference between the MApt difference for stimuli III and IV nor was it related to the difference between the aApt difference for these stimuli.

\subsection{Discussion}

The critical test for the variability of our paradigm was whether it could distinguish between the two convergent stimuli. To summarise the results, both the early and late kinematic measures reflected the difference in stimulus width between stimuli I and II. Responses to the thin convergent stimulus were different from responses to the thick convergent stimulus. This was most strongly reflected in the MApt differences. Furthermore, mean MApt to stimulus III was larger than to stimulus I, and mean MApt to stimulus IV was smaller than to stimulus II, whereas MApt did not differ between stimuli II and III, and between stimuli I and IV, showing that not only local, but also visible, global stimulus properties had an influence on the grasping movements under study. Moreover, the two kinematic measures aApt and MApt correlated positively. And the between-subjects variation was larger than the within-subjects variation, as reflected by the CoVs. This confirmed that the rank order of completion preferences from local to global was not caused by differences in within-subjects variation.

A potential objection to the correlations we found could be the large and consistent differences for two participants (19 and 3) at the one extreme and the two participants (11 and 12) at the other extreme. To address this point, we recalculated this analysis for all but the four participants at the extremes of the local-global spectrum. Removing participants from a dataset always affects the statistical power, and the correlation was indeed no longer present $(N=24, r=-3.257, p=0.12)$. However, the rationale behind the analyses was that participants in which the aApt reflected a strong preference for a specific type of completion, would show a similar preference as measured by the MApt. Considering this, we computed Spearman rank-order correlations that ignored the size of the parameter differences. Spearman's $\rho$ computed across all twenty-eight participants showed a positive trend $(N=28, \rho=0.335, p=0.08)$ but when we removed the four participants $3,11,19$, and 20 it proved still positive and significant $(N=24, \rho=0.419, p<0.05)$. Therefore, we argue that the four participants at the extremes of the spectrum were not outliers, and our claim, that a specific completion preference was reflected both in the early and late kinematic measure remains valid.

Before we discuss the results of our grasping experiment further, we first present the control experiment that was set up to test the relative completion preferences for the stimuli used in this study, and more specifically, to check the perceptual ambiguity of our divergent stimuli. 


\section{Perceptual control experiment}

\subsection{Method}

3.1.1 Participants. Twenty participants, eight male and twelve female, with ages ranging from 19 to 32 years (mean age, 23 years) participated in this experiment for remuneration or course credit after giving their informed consent. None of them had participated in the grasping experiment.

3.1.2 Material and design. We opted for a so-called speeded simultaneous-matching task to determine participants' preferences for completions of occlusion stimuli (eg Gerbino and Salmaso 1987; van Lier et al 1995b). The occlusion stimuli were identical to the images of the occluded cylindrical objects that were used as stimuli in the main experiment (see figures 1 and 7). The test shapes consisted of unoccluded cylindrical shapes. These cylindrical shapes were created by either linearly extending the partly occluded contours or by introducing an additional protrusion or indentation behind the occluder. For the divergent set this procedure resulted in local and global cylindrical objects, and for the convergent set this procedure resulted in either the simple cylindrical shapes (local and global convergent) or in irregular shapes (see inserts in figure 7). Stimuli were about $1.4 \mathrm{~cm}$ (wide segments) and $0.7 \mathrm{~cm}$ (narrow segments) wide and $19.5 \mathrm{~cm}$ high (visual angle: $12 \mathrm{deg}$ ). During the task, participants sat at a distance of about $80 \mathrm{~cm}$ behind a 19-inch computer monitor in a dimly lit room.

A timeline of the trial events of this experiment is shown in figure 6. Every trial started with a fixation cross in the centre of the screen, on which participants were instructed to focus. Then, a partly occluded shape (occlusion stimulus) was displayed $6.5 \mathrm{~cm}$ left from the centre for $500 \mathrm{~ms}$, after which an unoccluded test shape appeared next to the occlusion stimulus at $6.5 \mathrm{~cm}$ to the right side of the centre. The whole stimulus display with both occlusion stimulus and test shape was $19.5 \mathrm{~cm} \times 19.5 \mathrm{~cm}$. Participants were requested to judge whether the test shape could be identical to the occlusion stimulus or not, as soon as the test shape appeared. Participants responded by pressing a button on a button box that stood on the table in front of them. They were instructed to press the right ('yes') button with their right index finger when the test shape was a possible completion of the occlusion stimulus, and to press the left ('no') button with their left index finger when the test shape was not a possible completion of the occlusion stimulus. The button box had a $1 \mathrm{~ms}$ accuracy.

The test shape was a possible completion of the occlusion stimulus in half the trials (match trials), but not in the other half of the trials (no-match trials). The reaction times observed in the matching task provide information about the relative preferences of the different completions. While composing the non-match trials we followed the

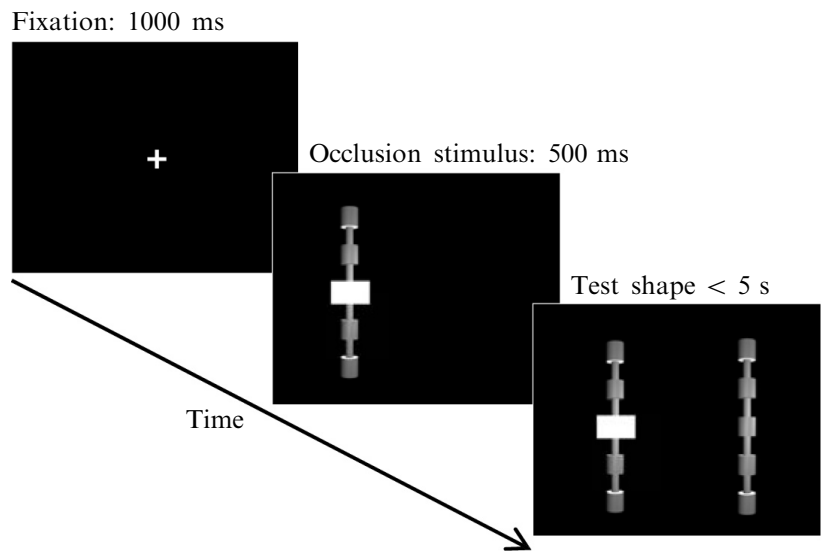

Figure 6. Trial events in the perceptual control experiment. 
same procedure as applied in Gerbino and Salmaso (1987) and van Lier et al (1995b). The no-match trials were composed such that occlusion stimuli were paired with completions of a different occlusion stimulus (eg occlusion stimulus III with the goal completion of stimulus IV). The relevant experimental conditions, ie the match trials, were composed as follows: trials could have occlusion stimuli that were convergent or divergent (stimulus type), and test shapes could be the result of a linear continuation of the partly occluded contours behind the occluder, or have an additional protrusion/indentation at that position (completion). As a result, test shapes for the divergent occlusion stimuli were either local or global completions, whereas test shapes for the convergent stimuli could be either simple cylinders or irregular shapes with an indentation or protrusion in the middle.

Each trial started with an occlusion stimulus presented for $500 \mathrm{~ms}$, after which a matching or non-matching test shape appeared next to the occlusion stimulus (see figure 6). All occlusion stimuli and corresponding test shapes of match-trials are shown in figure 7. Each combination of occlusion stimulus and test shape was replicated five times, resulting in a total of 80 trials for the whole experiment. Participants were given short breaks after the 30 th and the 60th trial. We expected shorter reaction times for the most preferred interpretation.

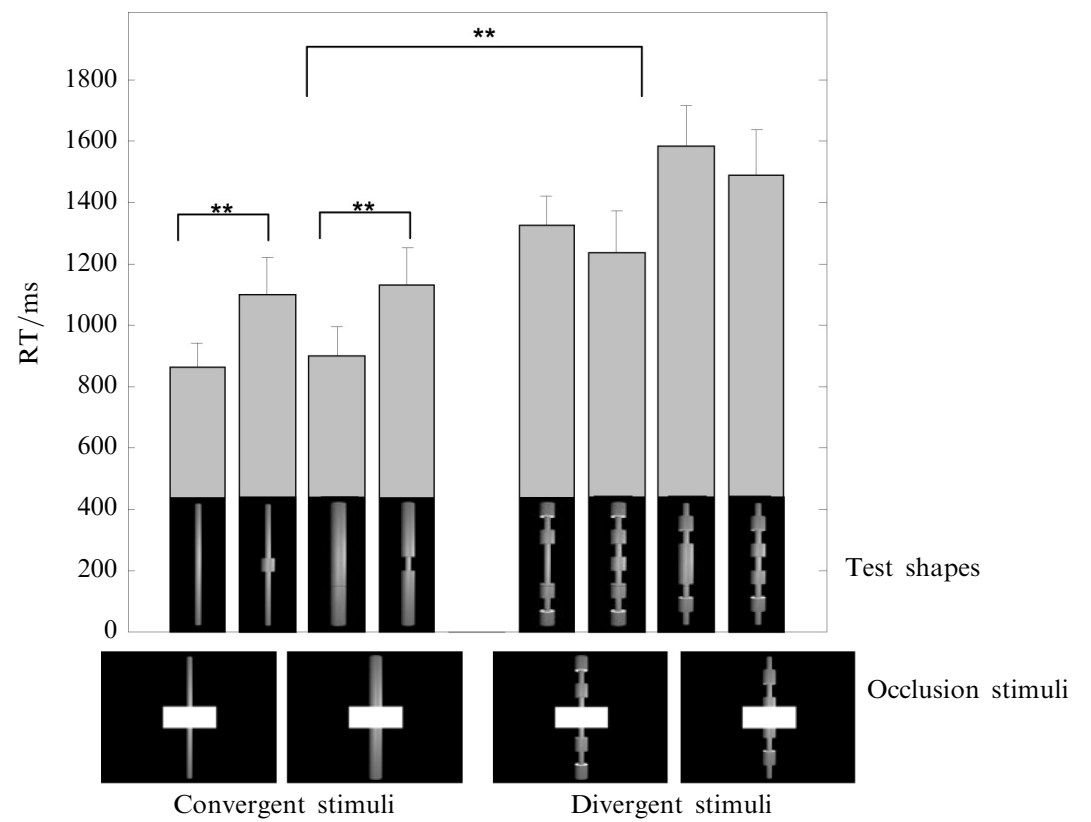

Figure 7. Reaction times for all convergent and divergent occlusion stimuli and test shapes in match trials, averaged across all participants. Every bar represents a reaction time (RT) averaged across the trials with the test stimulus that is shown at the base of each bar. Error bars indicate $\mathrm{SE}(* * p<0.01)$.

3.1.3 Data analysis. The main dependent variable in this task was the reaction time (RT) in the match trials with correct responses. RTs were compared between convergent and divergent stimuli and between local and global test stimuli, and evaluated by means of a $2 \times 2$ (stimulus type $\times$ completion) repeated-measures factorial ANOVA.

\subsection{Results}

We analysed only trials in which a correct response was given. On the basis of this criterion, $4 \%$ of all match trials were excluded. 
3.2.1 Reaction times. Overall RTs ranged from 366 to $4629 \mathrm{~ms}$, mean $=1198 \mathrm{~ms}$ $(\mathrm{SE}=25 \mathrm{~ms})$. Figure 7 shows RT for the two completion types for the convergent and divergent occlusion stimuli on all match trials. The $2 \times 2$ (stimulus type $\times$ completion) repeated-measures ANOVA showed that (i) responses to convergent stimuli ( $\mathrm{RT}=995 \mathrm{~ms}$, $\mathrm{SE}=29 \mathrm{~ms})$ were faster than responses to divergent stimuli $(\mathrm{RT}=1405 \mathrm{~ms}, \mathrm{SE}=39 \mathrm{~ms})$ $\left(F_{1,19}=55.982, p<0.01\right)$; (ii) no main effect of completion $\left(F_{1,19}=2.178\right.$, ns), and (iii) a significant interaction between stimulus type and completion $\left(F_{1,19}=16.769\right.$, $p<0.01)$.

Paired samples $t$-tests showed that, for the convergent stimuli, RTs were shorter in match trials with simple test shapes than in trials with irregular test shapes $\left(t_{19}=3.592, p<0.01\right)$, but there was no RT difference between the two divergent conditions $\left(t_{19}=-1.435\right.$, ns). Note that the RT difference between the two types of convergent test shapes confirms the sensitivity of the simultaneous matching paradigm.

Completion preferences for the divergent stimuli differed between participants. Twelve participants had on average shorter RTs in trials with global completions than in trials with local completions, whereas eight participants had on average shorter RTs in trials with local completions.

\subsection{Discussion}

The results of the control experiment confirmed our expectations. For the convergent stimuli, RTs were shorter for preferred completions. For the divergent stimuli, local and global completions did not yield statistically significant RT differences. In line with Gerbino and Salmaso (1987), and van Lier et al (1995b) this suggests that both forms of completion are about equally probable. This might explain why twelve participants preferred global completions and the other eight participants preferred local completions - a similar distribution as that observed in our grasping experiment.

\section{General discussion}

The goal of this study was to see if partly occluded stimuli differentially affect early or late kinematic measures of grasping movements. For this purpose we conducted an action experiment with perceptually ambiguous, partly occluded cylindrical stimuli, and an additional perceptual control experiment. In the action experiment, participants had to perform grasping movements in response to partly occluded cylindrical stimuli.

The results of the control experiment showed that, as expected, responses towards convergent trials were faster than responses towards divergent trials. The divergent stimuli resulted in longer processing time reflecting their greater complexity. Because partly occluded objects are often interpreted as wholes (Bruno et al 1997; Gerbino and Salmaso 1987; van Lier 1999; van Lier et al 1995b; Sekuler and Palmer 1992; de Wit and van Lier 2002), seeing the occlusion stimulus would facilitate recognition of the most plausible completed test shape. The comparison judgments can be made quicker if the test shapes are being recognised more easily. On the other hand, if the occlusion stimuli elicit both local and global interpretations that are about equally likely, then no RT difference is to be expected between the two divergent conditions. In general, the direction in the difference in response time between two completions is inversely related to the difference in preference (van Lier et al 1995b). In our control experiment, we found the expected RT differences for the convergent stimuli, and no overall difference in RTs between the global and local test shapes for the divergent stimuli.

The diameter of the target cylinder was not matched to the width of the visual stimuli. This way, participants knew that the target size was unrelated to the visual stimuli and they also knew that they grasped the same object time after time. Even despite this knowledge, the data of the grasping responses to the two convergent stimuli confirmed that our participants indeed followed the instructions "to grasp the target 
cylinder as if they grasped the visually presented stimulus behind the occluder". Although the participants grasped the same physical object in each trial, their grasping responses were influenced by the stimulus diameter. ${ }^{(2)}$ The analysis of the responses to the two convergent stimuli showed that the perceptual interpretations of these partly occluded stimuli were as predicted: significantly smaller aApt and MApt for the thin stimulus (stimulus I) as compared to the thick stimulus (stimulus II). This sensitivity demonstrated that our paradigm was an effective tool to study grasping movements to partly occluded stimuli.

Having established that our paradigm was viable, we can look at the influence ambiguous perceptual information has on early and late kinematics of the grasping responses. As we explained in section 1, local interpretations result from continuations of the contours behind the occluder and would lead to identical results for stimuli I and III and also for stimuli II and IV. Evidently, the experimental results show that this was not the case. Responses to stimulus III had on average a significantly larger MApt than responses to stimulus I, and responses to stimulus IV had on average a significantly smaller MApt than responses to stimulus II, whereas MApt did not differ between stimuli II and III and also not between stimuli I and IV. Therefore we can conclude that some process of perceptual integration of overall shape aspects took place, and that global stimulus properties did play an important role in establishing a representation of the stimulus that was used to guide the responses. It should be noted that an influence of the global stimulus properties implies that the general visible properties of the whole stimulus are taken into account to form a complete representation. Possibly, contributions of different types of visual information are weighted, depending on the competition van Lier et al (1995b) proposed.

The different kinematic profiles for the convergent and divergent stimuli show that the perceptual difference between the two stimulus categories affected grasping behaviour. That is, not only the width of the contours near the region of occlusion plays a role, but also the larger context of the stimuli, ie the overall regularity of the contours. Note that various computational completion models (Fantoni and Gerbino 2003; Kellman and Shipley 1991; Singh and Fulvio 2005) are based on local contour properties and do not take global properties (like overall symmetry, etc) of the partly occluded objects into account. Perceptual research has already stressed the importance of global stimulus attributes (van Lier et al 1994; de Wit et al 2006; de Wit and van Lier 2002), and now our grasping experiment shows that the susceptibility to global shape characteristics also affects the action domain. In daily life, such global object properties probably elicit top-down processes that enable us to recognise and act on objects around us.

In the trials with convergent stimuli, there is no reason to assume that a complex perceptual process interpreting the visual stimulus might have influenced the continuation of the grasping response after its initiation. Thus, the positive correlations between the two kinematic measures in the convergent condition indicate that it was correct to analyse aApt as an 'early' alternative for the more commonly used but relatively 'late' MApt. This is especially valuable in cases where one is specifically interested in processes

(2) It should be noted that the $0.4 \mathrm{~mm}$ difference between MApt in the two convergent conditions is slightly smaller than would be predicted on the basis of grasping responses towards real, non-occluded cylindrical targets of similar size. MApt for a grasping response towards a real non-occluded target would increase about 0.8 times with increasing target diameter (Marteniuk et al 1990; Smeets and Brenner 1999); in our experiment MApt increased only by a factor of 0.3 as a function of stimulus diameter. MApt in our task probably resulted from a combination of the size of the partly occluded cylinders, the size of the target cylinder, and the distance at which the stimuli were projected. Still, our grasping task has proven to be able to distinguish between grasping responses to stimuli of different widths. Also the MApt occurs a bit earlier (at 61\% of MT) as compared to normal grasping responses, but this moment was not affected by the stimulus conditions. 
that take place shortly after planning, and early on in the grasping response. The fact that we found positive correlations between aApt and MApt for both the convergent and divergent stimuli suggests that the movements of our participants were influenced by the same output of the perceptual process. Thus, ambiguity of the stimuli did not seem to have played a role during the grasping responses. The stable perceptual representation of the stimulus that was formed before response onset resulted in a plan for action that didn't change after response onset. Moreover, neither aApt nor MApt was bimodally distributed; thus we can be sure that the divergent stimuli were not represented in a perceptually bistable manner.

The positive correlations we found between the early and late kinematic measures proved to be independent from the initial ambiguity of the stimuli, despite the fact that the stimulus ambiguity resulted in opposing completion preferences across participants. One could argue that the local-global variations shown in figures 4 and 5 are normally distributed with means of approximately zero. However, the bottom panels of figures 4 and 5 show that, although the coefficients of variance varied remarkably between participants, the size of these coefficients was not related to the size of the MApt(stim III - stim IV) difference and aApt(stim III - stim IV) difference. This supports our idea that the contrasts we computed are not due to extreme variability in the response patterns of the participants with the most pronounced local or global responses patterns. The fact that the time delay of either $17 \mathrm{~ms}$ or $1000 \mathrm{~ms}$ between the presentation of the stimuli and the 'go' signal that prompted the grasping response, did not have any performance effects also shows that the amodal completion process yielded stable representations, the formation of which took less time than the prehension latencies.

The experimental setups of the control experiment and the grasping experiment differed primarily in terms of the visual angle at which the stimuli were presented. However, we did not set out to quantitatively compare the results of the two experiments but the control experiment was aimed to assess the strengths of preferences for both types of stimulus completion. Despite the differences between the two experiments, and the fact that a representation of a visual stimulus can be highly task-dependent, participants of both experiments showed qualitatively comparable behaviour that reflected the non-ambiguity of stimuli I and II and the ambiguity of stimuli III and IV.

In sum, we conducted a study of the effect of amodal completion on early and late kinematic features of grasping movements. The perceptual ambiguity of our stimuli was conveyed in the grasping data, and the results of both tasks showed an overall influence of global stimulus characteristics, but no overall preference for global or local completions, confirming the high degree of ambiguity of our stimuli. Moreover, the data of both experiments show that, with strongly ambiguous stimuli, completion preferences can differ remarkably between individuals, showing sensitivity to the overall shape and not just to the local creation of edges at points of occlusion. This is in contrast with theories of completion that focus on local contour properties (Fantoni and Gerbino 2003; Kellman and Shipley 1991). Thus, also with grasping movements towards a single object, in response to a partly occluded ambiguous stimulus, a stable perceptual representation of the earlier-seen stimulus is used, which is influenced by global stimulus properties.

\section{References}

Bruno N, Bertamini M, Domini F, 1997 "Amodal completion of partly occluded surfaces: Is there a mosaic stage?" Journal of Experimental Psychology: Human Perception and Performance 23 $1412-1426$

Fantoni C, Gerbino W, 2003 "Contour interpolation by vector - field combination” Journal of Vision $3281-303$

Gerbino W, Salmaso D, 1987 "The effect of amodal completion on visual matching" Acta Psychologica $\mathbf{6 5} 25-46$ 
Glover S, 2004 "Separate visual representations in the planning and control of action" Behavioural and Brain Sciences 27 - 78

Jakobson L S, Goodale M A, 1991 "Factors affecting higher-order movement planning: a kinematic analysis of human prehension" Experimental Brain Research 86 199-208

Jeannerod M, 1984 "The timing of natural prehension movements" Journal of Motor Behavior 16 235-254

Kellman P J, Shipley T F, 1991 "A theory of visual interpolation in object perception" Cognitive Psychology $23141-221$

Lier R J van, 1999 "Investigating global effects in visual occlusion: from a partly occluded square to the back of a tree-trunk" Acta Psychologica 120203 - 220

Lier R J van, Helm P A van der, Leeuwenberg E L J, 1994 "Integrating global and local aspects in visual occlusion" Perception $23883-903$

Lier R J van, Helm P A van der, Leeuwenberg E L J, 1995b "Competing global and local completions in visual occlusion" Journal of Experimental Psychology: Human Perception and Performance $21571-583$

Lier R J van, Leeuwenberg E L J, Helm P A van der, 1995a "Multiple completions primed by occlusion patterns" Perception 24727 - 740

Lier R J van, Wagemans J, 1999 "From images to objects: global and local completions of selfoccluded parts" Journal of Experimental Psychology: Human Perception and Performance $\mathbf{2 5}$ 1721 - 1741

Marteniuk R G, Leavitt J L, Mackenzie C L, Athenes S, 1990 "Functional relationships between grasp and transport components in a prehension task" Human Movement Science 9149 - 176

Meulenbroek R G J, Rosenbaum D A, Jansen C, Vaughan J, Vogt S, 2001 "Multijoint grasping movements - Simulated and observed effects of object location, object size, and initial aperture" Experimental Brain Research $138219-234$

Murray M M, Imber M L, Javitt D C, Foxe J J, 2006 "Boundary completion is automatic and dissociable from shape discrimination" Journal of Neuroscience 26 12043-12054

Sekuler A B, 1994 "Local and global minima in visual completion: effects of symmetry and orientation" Perception $23529-545$

Sekuler A B, Palmer S E, 1992 "Perception of partly occluded objects: A microgenetic analysis" Journal of Experimental Psychology: General 12195 - 111

Sekuler A B, Palmer S E, Flynn C, 1994 "Local and global processes in visual completion" Psychological Science 5 260-267

Singh M, Fulvio J M, 2005 "Visual extrapolation of contour geometry" Proceedings of the National Academy of Sciences of the USA $102939-944$

Smeets J B J, Brenner E, 1999 "A new view on grasping” Motor Control 3 237-271

Tse P U, 1999a "Complete mergeability and amodal completion" Acta Psychologica 102165 - 201

Tse P U, 1999b "Volume completion" Cognitive Psychology 39 37-68

Weigelt S, Singer W, Muckli L, 2007 "Separate cortical stages in amodal completion revealed by functional magnetic resonance adaptation" BMC Neuroscience 8 1- 11

Wit T C J de, Bauer M, Oostenveld R, Fries P, Lier R J van, 2006 "Cortical responses to contextual influences in amodal completion” NeuroImage 32 1815-1825

Wit T C J de, Lier R J van, 2002 "Global visual completion of quasi-regular shapes” Perception 31 $969-984$

Wouterlood D, Boselie F, 1992 "A good continuation model of some occlusion phenomena" Psychological Research $54267-277$ 


\section{PERTEPTION}

VOLUME 382009

www.perceptionweb.com

Conditions of use. This article may be downloaded from the Perception website for personal research by members of subscribing organisations. Authors are entitled to distribute their own article (in printed form or by e-mail) to up to 50 people. This PDF may not be placed on any website (or other online distribution system) without permission of the publisher. 